Edinburgh 2005/23

IFUM-868-FT

\title{
All order Running Coupling BFKL Evolution from GLAP (and vice-versa)
}

\author{
Richard D. Ball ${ }^{(a)}$ and Stefano Forte ${ }^{(b)}$ \\ ${ }^{(a)}$ School of Physics, University of Edinburgh \\ Edinburgh EH9 3JZ, Scotland \\ (b) Dipartimento di Fisica, Università di Milano and \\ INFN, Sezione di Milano, Via Celoria 16, I-20133 Milan, Italy
}

\begin{abstract}
We present a systematic formalism for the derivation of the kernel of the BFKL equation from that of the GLAP equation and conversely to any given order, with full inclusion of the running of the coupling. The running coupling is treated as an operator, reducing the inclusion of running coupling effects and their factorization to a purely algebraic problem. We show how the GLAP anomalous dimensions which resum large logs of $\frac{1}{x}$ can be derived from the running-coupling BFKL kernel order by order, thereby obtaining a constructive all-order proof of small $x$ factorization. We check this result by explicitly calculating the running coupling contributions to GLAP anomalous dimensions up to next-to-next-to leading order. We finally derive an explicit expression for BFKL kernels which resum large logs of $Q^{2}$ up to next-to-leading order from the corresponding GLAP kernels, thus making possible a consistent collinear improvement of the BFKL equation up to the same order.
\end{abstract}

December 2005 


\section{BFKL evolution and GLAP evolution}

The all-order summation of large logarithms of the center-of-mass energy in perturbative QCD is an intrinsically intricate problem, because it requires the simultaneous resummation of two large scales: the large energy, and the scale which renders the strong coupling perturbative. This difficulty is apparent when trying to fully include the perturbative running of the coupling in the evolution equation which sums energy logs in perturbative $\mathrm{QCD}$, the BFKL equation. In fact, doubts have been raised in the past on the consistency of a BFKL equation with running coupling. It is also apparent when trying to include a high-energy (i.e. small $x$ ) resummation in the standard renormalization-group (or GLAP) evolution equations of perturbative QCD: indeed, until recently it was not clear whether (beyond the leading-log level) a resummation of energy logs would be possible using the GLAP equation, without having to resort to the BFKL equation.

The perturbative expansion of the singlet GLAP splitting functions in powers of $\alpha_{s}\left(Q^{2}\right)$ at fixed $x$ is unstable at small $x$ : indeed, the recently computed NNLO corrections show explicitly that the instability is visible already around $x \sim 10^{-3}$. Analogously, the perturbative expansion of the BFKL kernel in powers of $\alpha_{s}\left(Q^{2}\right)$ at fixed $Q^{2}$ is unstable at large $Q^{2}$. In fact, as well known, this instability in practice makes the perturbative expansion of the BFKL kernel very poorly behaved essentially for all $Q^{2}$. Fortunately, it has now been demonstrated explicitly [1-6] that simultaneous resummation of the two large logs is feasible, and cures both instabilities. This double resummation can be accomplished in a transparent way by exploiting the duality [7-9] which relates the BFKL and GLAP equations, whereby one shows that, at the leading-twist level, both equations admit the same solutions and thus describe the same kind of evolution, provided their respective kernels are suitably matched.

Whereas at the fixed-coupling level this duality is straightforward to prove, it becomes rather nontrivial at the running coupling level. In fact, even the validity of runningcoupling factorization at small $x$ to any given logarithmic order (i.e. the existence of a universal evolution kernel which sums all pertinent logs for any given boundary condition) is nontrivial. Running coupling factorization and duality to any logarithmic order at small $x$ were first shown in Ref. [9]. The approach of that reference, however, besides being rather formal, was essentially geared to computation of small $x$ corrections to the GLAP equation, given the BFKL kernel as input. The inverse problem, namely the computation of large $Q^{2}$ corrections to the BFKL kernel given the GLAP kernel, though in principle possible, in practice requires an all-order computation. This computation is required in order to calculate stable BFKL kernels, and thus essential for any kind of phenomenology based on the BFKL equation. Furthermore, in the approach of Ref. [9] even the determination of next-to-next-to-leading $\ln \frac{1}{x}$ running-coupling corrections to the GLAP kernel obtained by fixed-coupling duality from BFKL is rather cumbersome.

In this paper, we will develop a rather general approach to running-coupling duality, based on the observation that the standard duality relations between GLAP and BFKL kernels, which are algebraic equations between kernels in a suitable double-Mellin transformed space, are promoted to operator equations when the coupling runs. We will show that these equations can be solved order-by-order by purely algebraic methods in terms of the commutators of the relevant operators, without having to actually determine their 
spectra, i.e. without having to solve high-order differential equations. This will enable us to determine running-coupling corrections to duality in either direction (i.e. when the BFKL kernel is determined from the GLAP one or conversely) and for either kind of logs. We will check the method and display its power by reproducing known results in a compact and efficient way, and then we will use it to obtain some new all-order results. As a byproduct, we will obtain a very direct re-derivation of factorization at small $x$.

The paper is organized as follows. In Sect. 3 we will define our notation, recall some basic results about duality at fixed and running coupling, and introduce the general formalism for the representation of running coupling evolution equations as operator equations. Then, in Sect. 4 we will derive within the operator approach the small $x$ resummation of the GLAP kernel from the BFKL kernel which was already discussed in Ref. [9]. We will then derive a compact expression, based on the Baker-Campbell-Hausdorff formula, which generates the corrections to fixed-coupling duality to any desired order (and with the running of the coupling given to any desired order). We will check it by reproducing the next-to-next-to-leading running coupling corrections to duality which in Ref. [10] could only be determined using computer algebra. Finally, in Sect. 5 we will construct the large $\ln Q^{2}$ resummation of the BFKL kernels from the GLAP kernels, which requires the computation of an infinite set of running-coupling terms: first, we will show that at the leading-log level there exists an operator ordering such that running-coupling duality relations reduced to the naive fixed-coupling ones, then, we will show that beyond the leading log level naive duality is modified by commutator terms, which we will determine explicitly up to the next-to-leading level. This will provide the first determination of a full collinear resummation of the next-to-leading-order BFKL kernel.

\section{Duality with fixed coupling and running coupling}

\subsection{General formalism}

We will discuss evolution equations for a parton distribution $G=x g\left(x, Q^{2}\right)$. This, according to the definition of the factorization scheme, can be thought of as the gluon density, or as an eigenvector of a two-by-two evolution matrix in the singlet sector. The kinematic variables $x$ and $Q^{2}$ can be thought of as the standard DIS variables, or more generally the perturbative scale and a dimensionless scale ratio such that $0 \leq x \leq 1$ : for example, for the production of a final state $X$ in hadronic collisions with center-of-mass energy $s$, $Q^{2} \equiv M_{X}^{2}$ (e.g. the Higgs mass), and $x \equiv Q^{2} / s$. We will not consider the dependence on other kinematic variables, such as transverse momentum and rapidity, i.e. we will consider standard parton distributions, for which ordinary collinear factorization applies.

In practice, it is convenient to express the parton density as a function of the logs of the relevant kinematic variables:

$$
G=G(\xi, t) ; \quad \xi \equiv \log \frac{1}{x}, \quad t \equiv \log \frac{Q^{2}}{\mu^{2}} .
$$

We define the Mellin transform with respect to either (or both) of the kinematic variables:

$$
G(N, t) \equiv \int_{0}^{\infty} d \xi e^{-N \xi} G(\xi, t)
$$




$$
G(\xi, M) \equiv \int_{-\infty}^{\infty} d t e^{-M t} G(\xi, t)
$$

Note that, by slight abuse of notation, we denote with the same symbol the parton distributions $G(N, t), G(\xi, M)$, and $G(N, M)$, although they are of course different functions of the respective arguments. In this paper we will mostly deal with the double-transformed distribution $G(N, M)$.

The GLAP equation takes the form

$$
\frac{d}{d t} G(N, t)=\gamma\left(\alpha_{s}(t), N\right) G(N, t)
$$

where $\alpha_{s}(t)$ is the running coupling, while the BFKL equation at the fixed coupling level takes the form

$$
\frac{d}{d \xi} G(\xi, M)=\chi\left(\alpha_{s}, M\right) G(\xi, M)
$$

If we expand the GLAP anomalous dimension $\gamma$ and the BFKL kernel $\chi$ in powers of $\alpha_{s}$ keeping the respective kinematic variable $(N$ or $M)$ fixed

$$
\begin{aligned}
\gamma\left(\alpha_{s}, N\right) & =\alpha_{s} \gamma_{0}(N)+\alpha_{s}^{2} \gamma_{1}(N)+\ldots \\
\chi\left(\alpha_{s}, M\right) & =\alpha_{s} \chi_{0}(M)+\alpha_{s}^{2} \chi_{1}(M)+\ldots,
\end{aligned}
$$

the GLAP equation (2.4) sums $\mathrm{N}^{k+1} \mathrm{LO} \ln Q^{2}$ terms if contributions up to $\gamma_{k}$ are included, and the BFKL equation (2.5) sums $\mathrm{N}^{k+1} \mathrm{LO} \ln \frac{1}{x}$ terms if contributions up to $\chi_{k}$ are included in the respective kernels.

The fixed coupling duality relation states that if kernels $\chi$ and $\gamma$ related by

$$
\begin{aligned}
& N=\chi\left(\alpha_{s}, \gamma\left(\alpha_{s}, N\right)\right), \\
& M=\gamma\left(\alpha_{s}, \chi\left(\alpha_{s}, M\right),\right.
\end{aligned}
$$

then the BFKL and GLAP equation admit the same solution. This relation is straightfoward to derive $[1,8]$ from the observation that the leading twist behaviour of $G(N, M)$ is

determined by its pole in the $M, N$ plane, and that eqs. (2.8),(2.9) express the position of this pole.

\subsection{Running coupling duality}

At the running coupling level, the BFKL kernel becomes an operator in $M$ space. Indeed, the $\left(x, Q^{2}\right)$ BFKL equation, obtained by inverse Mellin transform from eq. (2.5) has the form

$$
\frac{d}{d \xi} G\left(\xi, Q^{2}\right)=\int_{-\infty}^{\infty} \frac{d k^{2}}{k^{2}} \sum_{n=0}^{\infty} \alpha_{s}^{n} K_{n}\left(Q^{2} / k^{2}\right) G\left(\xi, k^{2}\right),
$$

where

$$
\chi_{n}(M)=\int_{0}^{\infty} \frac{d Q^{2}}{Q^{2}}\left(\frac{Q^{2}}{k^{2}}\right)^{-M} K_{n}\left(\frac{Q^{2}}{k^{2}}\right) .
$$


Therefore, if in eq. (2.11) we let the coupling run with $Q^{2}, \alpha_{s} \rightarrow \alpha_{s}\left(Q^{2}\right)$, upon Mellin transformation we get

$$
\frac{d}{d \xi} G(\xi, M)=\sum_{n=0}^{\infty} \hat{\alpha}_{s}^{n} \chi_{n}(M) G(\xi, M),
$$

where $\hat{\alpha}_{s}$ is the operator obtained by the replacement $t \rightarrow-\frac{\partial}{\partial M}$ in the expression for $\alpha_{s}\left(Q^{2}\right)$. For example, at the leading log level, where $\alpha_{s}\left(Q^{2}\right)=\alpha_{s} /\left(1+\beta_{0} \alpha_{s} t\right)$ we get

$$
\widehat{\alpha}_{s}=\frac{\alpha_{s}}{1-\beta_{0} \alpha_{s} \frac{\partial}{\partial M}}
$$

where $\alpha_{s} \equiv \alpha_{s}\left(\mu^{2}\right)$. Note that this implies

$$
\left[\hat{\alpha}_{s}^{-1}, M\right]=-\beta_{0}
$$

If we start again with eq. (2.10), but we let $\alpha_{s} \rightarrow \alpha_{s}\left(k^{2}\right)$, upon inverse Mellin transform we get instead

$$
\frac{d}{d \xi} G(\xi, M)=\sum_{n=0}^{\infty} \chi_{n}(M) \hat{\alpha}_{s}^{n} G(\xi, M) .
$$

Of course, in general $\hat{\alpha}_{s}$ and $\chi_{n}(M)$ do not commute, and therefore the explicit form of the functions $\chi_{n}(M)$ will depend on the argument of the coupling. However, given $\chi_{n}(M)$ which correspond to one ordering, those which correspond to a different ordering can be determined by simply computing the relevant commutators. Of course, this is just the $M$-space form of the trivial observation that given the expansion of the kernel $K\left(\alpha_{s}\left(Q^{2}\right), Q^{2} / k^{2}\right)$ in powers of $\alpha_{s}\left(Q^{2}\right)$ we may determine its expansion in terms of $\alpha_{s}\left(k^{2}\right)$ by expressing $\alpha_{s}\left(Q^{2}\right)$ in terms of $\alpha_{s}\left(k^{2}\right)$ through the renormalization-group equation. Therefore, at the runnning coupling level the BFKL kernel $\chi\left(\hat{\alpha}_{s}, M\right)$ is an operator, and its explicit form depends on the operator ordering. Note that if the evolution equations are written in double-Mellin $(M, N)$ space, the GLAP anomalous dimension also becomes the operator $\gamma\left(\hat{\alpha}_{s}, N\right)$ obtained by replacing $\alpha_{s}(t) \rightarrow \hat{\alpha}_{s}$ in eq. (2.6), but since $\hat{\alpha}_{s}$ commutes with a function of $N$ the explicit form of $\gamma\left(\hat{\alpha}_{s}, N\right)$ does not depend on operator ordering.

We will assume henceforth for definiteness that $\alpha_{s}$ runs with $Q^{2}$. In such case, the running coupling GLAP and BFKL equations in $(M, N)$ space are

$$
\begin{aligned}
M G(N, M) & =\gamma\left(\hat{\alpha}_{s}, N\right) G(N, M)+G_{0}(N) \\
N G(N, M) & =\chi\left(\hat{\alpha}_{s}, M\right) G(N, M)+F_{0}(M),
\end{aligned}
$$

where $\gamma\left(\hat{\alpha}_{s}, N\right)$ and $\chi\left(\hat{\alpha}_{s}, M\right)$ are respectively defined by the expansions eq. (2.6),(2.7) with $\alpha_{s} \rightarrow \hat{\alpha}_{s}$ (and all powers of $\hat{\alpha}_{s}$ to the left), and $G_{0}$ and $F_{0}$ are boundary conditions. Running coupling duality is the statement that given $\chi$ there exists a $\gamma$ such that the solutions to eqs. $(2.16),(2.17)$, when transformed back to $\left(Q^{2}, x\right)$ space, coincide at leading twist, and conversely given $\gamma$ there exists a $\chi$ with this property. This result is highly nontrivial in that it relates solutions to high-order differential equations. 
With the leading-order form of $\hat{\alpha}_{s}$ eq. (2.13), we can formally expand the runningcoupling GLAP and BFKL equations in powers of $\beta_{0}$. This generates a series of runningcoupling corrections to fixed coupling duality, which we can compute order by order by solving both equations iteratively as a series in powers of $\beta_{0}$, and then matching the solutions. The fact that this matching is possible is nontrivial, however, because if we start, say, from BFKL and determine the dual GLAP kernel, the $Q^{2}$ dependence of the solutions must match exactly (up to higher-twist corrections), i.e. to all orders in an expansion in powers of $t$ up to the given order in $\beta_{0}$. So, for instance, at $O\left(\beta_{0}^{2}\right)$ the solution contains terms up to $O\left(t^{2}\right)$ in an expansion in powers of $t$, which must all be matched in terms of a single $O\left(\beta_{0}^{2}\right)$ correction to the duality relation between kernels.

In Refs. $[8,10]$ it was explicitly checked that this matching is possible up to order $\beta_{0}^{2}$, and the corresponding running duality corrections were explicitly given for the determination of the GLAP anomalous dimension from the BFKL kernel. The nontrivial nature of this matching suggested that running duality should in fact hold to all orders. In Ref. [9] it was shown that this is indeed the case to all orders in $\beta_{0}$, if one uses the leading-order form eq. (2.13) of the running coupling, and the leading-order expression of $\chi$ eq. (2.7), i.e., if the BFKL kernel is linear in $\hat{\alpha}_{s}$. Indeed, in this case it is possible to solve the running-coupling $(M, N)$-space BFKL equation exactly as a differential equation. It can then be proven that in the large $Q^{2}$ limit only the inhomogeneous solution to this differential equation survives, which is linear in the boundary condition. Furthemore, its $Q^{2}$-dependence can be determined by saddle-point and shown to exponentiate in terms of an anomalous dimension which is thereby determined order-by-order in $\beta_{0}$ by the saddle point expansion, and seen to reproduce the perturbative result.

Even though it is reasonable to conjecture that these results are quite general, so that factorization and duality hold in fact to any logarithmic order, no general argument is available. Furthermore, the saddle-point method of ref. [9] is only applicable if $\chi$ is linear in $\hat{\alpha}_{s}$ and the latter has the leading-order form eq. (2.13). The perturbative brute-force matching can of course be applied systematically, but it rapidly becomes computationally very cumbersome: in fact, even the computation of $O\left(\beta_{0}^{2}\right)$ corrections to duality in ref. [10] could only be accomplished by means of computer algebra. Here we will present an approach which is entirely general and computationally much simpler, even though of course still amenable to computer implementation.

\section{GLAP leading singularities from BFKL kernels}

As apparent from eq. (2.13), the running of the coupling is a leading $\ln Q^{2}$ effect, but it is subleading $\ln \frac{1}{x}$, and in fact whereas at leading $\ln Q^{2}$ terms to all orders in $\alpha_{s} \beta_{0}$ contribute, to next ${ }^{n}$-to-leading $\ln \frac{1}{x}$ only terms up to $O\left(\left(\alpha_{s} \beta_{0}\right)^{n}\right)$ should be included. Hence, the determination of running coupling corrections to duality is simpler when computing the leading and subleading $\ln \frac{1}{x}$ corrections to GLAP splitting functions from BFKL kernels than when computing leading and subleading $\ln Q^{2}$ corrections to BFKL kernels from GLAP splitting functions: in the former case, the number of terms which must be determined to each logarithmic order is finite, while it is infinite in the latter case. In this section we consider the simpler problem, postponing the more difficult case to the next section. 
The problem is to start from eq. (2.17), with the BFKL kernel $\chi$ computed to some finite order in the expansion eq. (2.7), and determine whether there exists a function $\gamma\left(\alpha_{s}, N\right)$ such that, if one uses it in the GLAP equation (2.16) the solutions to the two equations coincide at leading twist. In the fixed coupling case, the solution is given by eqs. (2.8),(2.9), which in particular imply that if terms up to order $\alpha_{s}^{n}$ are included in the expansion (2.7), then $\gamma$ has the form

$$
\gamma\left(\alpha_{s}, N\right)=\gamma_{s}\left(N / \alpha_{s}\right)+\alpha_{s} \gamma_{s s}\left(N / \alpha_{s}\right)+\ldots
$$

In the running coupling case, the problem was solved perturbatively in Ref. [8]. Consider for definiteness the case in which we only include the leading-order contribution $\chi_{0}$ to the expansion of the BFKL kernel $\chi$ eq. (2.7), and we let $\alpha_{s} \rightarrow \hat{\alpha}_{s}$ eq. (2.13). The perturbative solution then proceeds in four steps: first, $\hat{\alpha}_{s}$ Eq. (2.13) is expanded as a power series in $\alpha_{s} \beta_{0} \frac{\partial}{\partial M}$ and the running-coupling BFKL equation (2.17) is solved perturbatively, with a result of the form

$$
G(N, M)=\left[\frac{1}{1-\frac{\alpha_{s}}{N} \chi_{0}}+\alpha_{s} \frac{\alpha_{s}}{N}\left(\frac{\beta_{0} \chi_{0}\left(\ln \chi_{0} G_{0}\right)^{\prime}}{\left(1-\frac{\alpha_{s}}{N} \chi_{0}\right)^{2}}+\frac{\beta_{0} \chi_{0} \frac{\alpha_{s}}{N} \chi_{0}^{\prime}}{\left(1-\frac{\alpha_{s}}{N} \chi_{0}\right)^{3}}\right)+\ldots\right] \frac{G_{0}(M)}{N} .
$$

Second, the inverse $M$-Mellin transform $G(N, t)$ of this solution is determined up to the desired order. Third, this solution is compared to the expansion of the solution to the $(N, t)$ space GLAP equation (2.4) to the same order in $\alpha_{s}$ and linear in $t$, and the corresponding $\gamma$ is determined, as the sum of $\gamma$ determined at the next-lowest order, and a running-coupling correction. Finally, it is verified that terms corresponding to all higher order powers of $t$ which appear at the given order in $\alpha$ exponentiate, so that the solution to Eq. (2.4) is fully reproduced to the given order.

The exponentiation was verified explicitly up to next-to-next-to leading order in refs. [9,10], and the running coupling anomalous dimension was found to be $\gamma\left(\alpha_{s}(t), N\right)$, where

$$
\gamma\left(\alpha_{s}, N\right)=\gamma_{s}\left(N / \alpha_{s}\right)+\alpha_{s} \beta_{0} \Delta \gamma_{s s}\left(N / \alpha_{s}\right)+\left(\alpha_{s} \beta_{0}\right)^{2} \Delta \gamma_{s s s}\left(N / \alpha_{s}\right)+O\left(\alpha_{s} \beta_{0}\right)^{3},
$$

with $\gamma_{s}\left(N / \alpha_{s}\right)$ determined by fixed coupling duality eq. (2.8), i.e. as the solution of

$$
N=\alpha_{s} \chi_{0}\left(\gamma_{s}\left(N / \alpha_{s}\right)\right)
$$

and

$$
\begin{aligned}
\Delta \gamma_{s s}\left(N / \alpha_{s}\right) & =-\left.\chi_{0} \frac{\chi_{0}^{\prime \prime}}{2 \chi_{0}^{\prime 2}}\right|_{M=\gamma_{s}\left(N / \alpha_{s}\right)} \\
\Delta \gamma_{s s s}\left(N / \alpha_{s}\right) & =-\left.\chi_{0}^{2} \frac{15 \chi_{0}^{\prime \prime 3}-16 \chi_{0}^{\prime} \chi_{0}^{\prime \prime} \chi_{0}^{\prime \prime \prime}+3{\chi_{0}^{\prime}}^{2} \chi_{0}^{\prime \prime \prime \prime}}{24 \chi_{0}^{\prime 5}}\right|_{M=\gamma_{s}\left(N / \alpha_{s}\right)},
\end{aligned}
$$

where all derivatives are with respect to the argument of $\chi_{0}(M)$, which is then evaluated at $\gamma_{s}\left(N / \alpha_{s}\right)$. 
We can view these running coupling terms as a modification of $\chi$, which gets replaced by $\chi^{\text {eff }}\left(\hat{\alpha}_{s}, M\right)=\hat{\alpha}_{s} \chi_{0}(M)+\hat{\alpha}_{s}^{2}\left(\chi_{1}(M)+\Delta \chi_{1}(M)\right)+\ldots$ from which $\gamma$ eq. (3.3) is obtained using naive duality eq. (2.8), with

$$
\Delta \chi_{1}=\beta_{0} \frac{\chi_{0}^{\prime \prime} \chi_{0}}{2 \chi_{0}^{\prime}}
$$

and so on. Of course, if higher order contributions to $\chi$ eq. (2.7) are included, they also contribute to $\gamma_{s}, \gamma_{s s}$, etc., as dictated by fixed-coupling duality.

This next-to-next-to-leading order exponentiation was shown to follow from an allorder argument in ref. [9], were the next-to-leading correction was also rederived as a byproduct through a different, though hardly easier method, based on an asymptotic expansion of the exact solution of the running coupling BFKL equation (2.17). Here we will show that these results can be obtained in a much simpler and general algebraic setting without having to actually solve the running coupling BFKL and GLAP equations, either perturbatively or exactly.

\subsection{A model calculation}

Let us start by considering a toy calculation where the GLAP kernel is given by its leading singularity, i.e. $\gamma\left(\hat{\alpha}_{s}, N\right)=N^{-1} \hat{\alpha}_{s}$. In this case, the GLAP eq. (2.16) reduces to

$$
\left(M-N^{-1} \hat{\alpha}_{s}\right) G(N, M)=G_{0}(N),
$$

which, multiplying by $N M^{-1}$ on the left and noting that $\left[N, \hat{\alpha}_{s}\right]=[N, M]=0$ reduces to

$$
\left(N-M^{-1} \hat{\alpha}_{s}\right) G(N, M)=M^{-1} N G_{0}(N) .
$$

Recalling $[1,8]$ that the large $Q^{2}$ behaviour of the solution is determined by the pole in the $M, N$ plane, this is seen to reduce to the BFKL-like equation

$$
N G(N, M)=M^{-1} \hat{\alpha}_{s} G(N, M)+F_{0}(M),
$$

with a suitably readjusted boundary condition $F_{0}(M)=M^{-2} \hat{\alpha}_{s} G_{0}\left(M^{-1} \hat{\alpha}_{s}\right)$. Hence, the running dual of $\gamma=N^{-1} \hat{\alpha}_{s}$ is

$$
\begin{aligned}
\chi\left(\hat{\alpha}_{s}, M\right) & =M^{-1} \hat{\alpha}_{s}=\hat{\alpha}_{s} M^{-1}+\beta_{0} M^{-1} \hat{\alpha}_{s}^{2} M^{-1} \\
& =\hat{\alpha}_{s} M^{-1}+\beta_{0} \hat{\alpha}_{s}^{2} M^{-2}+O\left(\hat{\alpha}_{s}^{3}\right),
\end{aligned}
$$

where we have used the commutator

$$
\left[\hat{\alpha}_{s}, M^{-1}\right]=-\beta_{0} \hat{\alpha}_{s} M^{-2} \hat{\alpha}_{s}=-\beta_{0} M^{-1} \hat{\alpha}_{s}^{2} M^{-1},
$$

which may be readily obtained from eq. (2.14).

Now we note that the $O\left(\hat{\alpha}_{s}\right)$ term is just the fixed-coupling dual of $\gamma$, while the $O\left(\hat{\alpha}_{s}^{2}\right)$ term coincides with minus the running coupling correction eq. (3.7): with $\chi_{0}=1 / M$, 
$\frac{\chi_{0}^{\prime \prime} \chi_{0}}{2 \chi_{0}^{\prime}}=-1 / M^{2}$. This is as it should be, because Eq. (3.5) gives the addition to $\gamma$ due to the running-coupling corrections which are necessary for it to be the running coupling dual of a given $\chi$. Equation (3.7) then expresses this quantity as an effective contribution to $\chi$. Equation (3.11) instead gives the $\chi$ which we must start from in order to obtain $\gamma=\alpha_{s} / N$ through running-coupling duality. Clearly, this $\chi$ is obtained by undoing the effect of running duality, i.e. by starting off with a $\chi$ which differs from the naive dual by a term which is then removed by the running coupling correction.

We conclude that in this simple case we have in fact reproduced the running-coupling correction to duality. We notice furthermore that the boundary condition did not play any role in the argument: in fact, the above argument can be recast as the simple observation that the operator equation

$$
N G(N, M)=M^{-1} \hat{\alpha}_{s} G(N, M)
$$

can be rewritten as

$$
M G(N, M)=N^{-1} \hat{\alpha}_{s} G(N, M) .
$$

The running-coupling duality corrections are then simply due to the commutators which are necessary in order to bring the the BFKL kernel in the form of eq. (2.12), which in this simple example are straightforward to compute.

In the next subsection we shall prove that in fact the determination of running coupling duality corrections can always be reduced to the algebraic manipulation of an operator equation, which we will then perform systematically in the subsequent section.

\subsection{Factorization}

In order to discuss the form of the duality condition, it is more convenient to start from the GLAP equation (2.16), which can be formally solved as

$$
G(N, M)=\left(M-\gamma\left(\hat{\alpha}_{s}, N\right)\right)^{-1} G_{0}(N) .
$$

This equation formally embodies all order (GLAP) factorization, in the sense that on the right hand side the perturbative evolution factorises from the nonperturbative boundary condition $G_{0}(N)$. We can expand this solution about any $\bar{N}$ as

$G(N, M)=\left[M-\gamma\left(\hat{\alpha}_{s}, \bar{N}\right)-\gamma^{\prime}\left(\hat{\alpha}_{s}, \bar{N}\right)(N-\bar{N})+O\left((N-\bar{N})^{2}\right)\right]^{-1}\left(G_{0}(\bar{N})+O(N-\bar{N})\right)$

where the prime denotes partial differentiation with respect to $N$.

Now, the leading twist behaviour of $G(N, t)$ at large $t$ is given by the pole in the $M$-plane due to the vanishing of the denominator in (3.15): at the pole

$$
M G(N, M)=\gamma\left(\hat{\alpha}_{s}, N\right) G(N, M) .
$$

Clearly the position of the pole will depend on $N$. Now assume that there exists an operator-valued function $\chi\left(\hat{\alpha}_{s}, M\right)$ such that at the pole eq. (3.17) may be inverted, i.e.

$$
N G(N, M)=\chi\left(\hat{\alpha}_{s}, M\right) G(N, M)
$$


The operator $\chi\left(\hat{\alpha}_{s}, M\right)$ is the inverse of the operator $\gamma\left(\hat{\alpha}_{s}, N\right)$, in the sense that

$$
M G(N, M)=\gamma\left(\hat{\alpha}_{s}, \chi\left(\hat{\alpha}_{s}, M\right)\right) G(N, M),
$$

and conversely

$$
N G(N, M)=\chi\left(\hat{\alpha}_{s}, \gamma\left(\hat{\alpha}_{s}, N\right)\right) G(N, M)
$$

Of course the existence of the function $\chi\left(\hat{\alpha}_{s}, M\right)$ is nontrivial: we will show how it may be constructed order by order in the next section.

Given $\chi\left(\hat{\alpha}_{s}, M\right)$, we may then choose $\bar{N}$ in the expansion (3.16) to be the position of the perturbative pole in the $N$-plane for a given $M$ : using (3.19) the first two terms in the square brackets then cancel by construction, and we are left with

$$
\left.G(N, M)=\left(N-\chi\left(\hat{\alpha}_{s}, M\right)\right)\right)^{-1}\left(-\gamma^{\prime}\left(\hat{\alpha}_{s}, \chi\left(\hat{\alpha}_{s}, M\right)\right)\right)^{-1} G_{0}\left(\chi\left(\hat{\alpha}_{s}, M\right)\right)+\ldots,
$$

where the dots denote terms which are regular in the neighbourhood of the pole. We may then define the function

$$
F_{0}(M)=\left(-\gamma^{\prime}\left(\hat{\alpha}_{s}, \chi\left(\hat{\alpha}_{s}, M\right)\right)\right)^{-1} G_{0}\left(\chi\left(\hat{\alpha}_{s}, M\right)\right),
$$

which is just some function of $M$, related in a complicated way to the original function $G_{0}(M)$, but with manifestly no dependence on $N$. Identifying $F_{0}(M)$ with a BFKL boundary condition, eq. (3.21) is the solution to the running coupling BFKL equation (2.17), with the kernel $\chi\left(\hat{\alpha}_{s}, M\right)$ given by eq. (3.19). The solution is again factorised at the pole into a perturbative evolution factor and a nonperturbative boundary condition. Thus all orders factorization of the running coupling BFKL equation follows directly from all orders factorization of GLAP provided only that the kernel defined through eq. (3.19) actually exists.

It is important to understand in which respect eq. (3.19) differs from the naive fixed coupling duality relation eq. (2.9), to which it bears formal resemblance. The meaning of this equation is that at the pole, the operators $N$ and $\chi\left(\hat{\alpha}_{s}, M\right)$ have the same action on the physical solution $G(N, M)$, even though they are in general different operators. The anomalous dimension $\gamma\left(\hat{\alpha}_{s}, N\right)$ is then the operator such that if we apply it to a physical state at the pole, and then use repeatedly eq. (3.18), we obtain the 1.h.s. of eq. (3.19), i.e. $M$. Just as in the fixed coupling case the duality relation (3.19) is invertible: it can be used to construct $\chi$ given $\gamma$ or $\gamma$ given $\chi$.

To make this more explicit, consider the simple example of sect. 3.1. Starting with $\gamma=$ $N^{-1} \hat{\alpha}_{s}$, the pole condition (3.17) becomes $M G=N^{-1} \hat{\alpha}_{s} G=\hat{\alpha}_{s} N^{-1} G$. Then construct $\chi$ by demanding that it satisfies (3.19):

$$
M G=\hat{\alpha}_{s}\left(\chi\left(\hat{\alpha}_{s}, M\right)\right)^{-1} G .
$$

Note the ordering here: only when the factor of $N^{-1}$ is on the right, and thus able to act on $G$, can we use (3.18). Expanding $\chi$ at lowest nontrivial order we get

$$
\begin{aligned}
M G & =\left[\hat{\alpha}_{s}\left(\left(\hat{\alpha}_{s} \chi_{0}(M)+\hat{\alpha}_{s}^{2} \chi_{1}\right)+\cdots\right)^{-1}\right] G \\
& =\left[\hat{\alpha}_{s}\left(\chi_{0}(M)+\hat{\alpha}_{s} \chi_{1}(M)+\cdots\right)^{-1} \hat{\alpha}_{s}^{-1}\right] G \\
& =\left[\left(\chi_{0}(M)+\hat{\alpha}_{s} \chi_{1}(M)+\cdots\right)^{-1}-\hat{\alpha}_{s} \beta_{0} \frac{\chi_{0}^{\prime}(M)}{\chi_{0}^{2}(M)}\right] G,
\end{aligned}
$$


where in the last line we used the commutator

$$
\left[\hat{\alpha}_{s}^{-1}, \chi_{0}^{-1}(M)\right]=-\beta_{0}\left(\chi_{0}^{-1}(M)\right)^{\prime}=\beta_{0} \chi_{0}^{\prime} / \chi_{0}^{2}
$$

We immediately get that to order $\hat{\alpha}_{s}, \chi_{0}(M)=1 / M$, and substituting this back in eq. (3.24) and expanding $\chi_{1}(M)=\beta_{0} / M^{2}$, in agreement with eq. (3.11).

Hence, we see that the running coupling dual is determined by solving either of the two operator equations $(3.20),(3.19)$ from the respective pole conditions $(3.17),(3.18)$. In the next subsection, we will construct a solution of these equations which, when expanded out, generates running coupling corrections to any desired order.

\subsection{A Systematic Algebraic approach}

The construction of the running coupling dual of a given BFKL kernel or GLAP anomalous dimension presented in the previous subsection can be formalized in the following way: given an operator equation of the form

$$
\hat{p} G=\hat{q} G
$$

(where $G$ denotes the physical solution), and given a function $f(\hat{q})$, determine the function $g$ such that using eq. (3.26) on physical solutions one gets

$$
f(\hat{q}) G=g(\hat{p}) G
$$

Note that $f \neq g$ necessarily, since $\hat{p}$ and $\hat{q}$ do not commute: for example

$$
\hat{q}^{2} G=\hat{q} \hat{p} G=(\hat{p} \hat{q}+[\hat{q}, \hat{p}]) G=\left(\hat{p}^{2}+[\hat{q}, \hat{p}]\right) G \neq \hat{p}^{2} G
$$

if $[\hat{q}, \hat{p}] \neq 0$. So the problem is in general nontrivial.

Equation (3.18) corresponds to the particular choice

$$
\begin{aligned}
& \hat{p}=\hat{\alpha}_{s}^{-1} N \\
& \hat{q}=\hat{\alpha}_{s}^{-1} \chi\left(\hat{\alpha}_{s}, M\right),
\end{aligned}
$$

where for future convenience we have multiplied both sides by $\hat{\alpha}_{s}$. Equation (3.17) corresponds instead to the choice

$$
\begin{aligned}
& \hat{p}=M \\
& \hat{q}=\gamma\left(\hat{\alpha}_{s}, N\right) .
\end{aligned}
$$

In the latter case, for example, we can choose the function $f=\tilde{\chi}$, such that $\tilde{\chi}\left(\gamma\left(\hat{\alpha}_{s}, N\right)\right)=$ $N$ : because $\hat{\alpha}_{s}$ and $N$ commute this is just the naive dual. Equation (3.27) then determines the running coupling dual $\chi(M)$ as the function $g(\hat{p})$.

A general solution to this problem may be obtained by using the Baker-CampbellHausdorff formula for a pair of noncommuting operators $A$ and $B$ which to cubic order is [11]

$$
e^{A} e^{B}=\exp \left\{A+B+\frac{1}{2}[A, B]+\frac{1}{12}([A,[A, B]]+[B,[B, A]])+\ldots\right\} .
$$


Letting $A=\hat{q}$ and $B=\hat{p}-\hat{q}$ we get

$e^{\hat{q}} e^{\hat{p}-\hat{q}}=\exp \left\{\hat{p}-\frac{1}{2}[\hat{p}, \hat{q}]+\frac{1}{6}[\hat{q},[\hat{q}, \hat{p}]]+\frac{1}{12}[\hat{p},[\hat{p}, \hat{q}]]+\frac{1}{24}[\hat{q},[\hat{q},[\hat{q}, \hat{p}]]]+\frac{1}{24}[\hat{q},[\hat{p},[\hat{p}, \hat{q}]]]+\ldots\right\}$.

Multiplying the right-hand side by the identity $e^{\hat{p}} e^{-\hat{p}}=1$ on the left, and using the BakerCampbell-Hausdorff formula again with $A=-\hat{p}$ and $B$ set equal to the exponent of the exponential on the r.h.s., eq. (3.31) becomes

$$
\begin{aligned}
e^{\hat{q}} e^{\hat{p}-\hat{q}}=e^{\hat{p}} \exp \left\{-\frac{1}{2}[\hat{p}, \hat{q}]+\frac{1}{6}[\hat{q},[\hat{q}, \hat{p}]]+\frac{1}{3}[\hat{p},[\hat{p}, \hat{q}]]+\frac{1}{24}[\hat{q},[\hat{q},[\hat{q}, \hat{p}]]]\right. & \\
& \left.+\frac{1}{8}[\hat{q},[\hat{p},[\hat{p}, \hat{q}]]]-\frac{1}{8}[\hat{p},[\hat{p},[\hat{p}, \hat{q}]]]+\ldots\right\}
\end{aligned}
$$

Now, eq. (3.26) implies that

$$
e^{\hat{p}-\hat{q}} G=G,
$$

so eq. (3.32) gives us an expression for the action of $e^{\hat{q}}$ on physical solutions $G$. We can use this result to construct a general solution to our problem by noting that any function $f(\hat{q})$ can be constructed by Taylor expansion as

$$
f(\hat{q})=\left.e^{\hat{q} \frac{d}{d \lambda}} f(\lambda)\right|_{\lambda=0} .
$$

Rescaling the operators $\hat{p}$ and $\hat{q}$ in eq. (3.32) by $\frac{d}{d \lambda}$, i.e. letting $\hat{p} \rightarrow \hat{p} \frac{d}{d \lambda}, \hat{q} \rightarrow \hat{q} \frac{d}{d \lambda}$ we get finally

$$
\begin{aligned}
f(\hat{q}) G=e^{\hat{p} \frac{d}{d \lambda}} \exp \{ & -\frac{1}{2}[\hat{p}, \hat{q}] \frac{d^{2}}{d \lambda^{2}}+\frac{1}{6}[\hat{q},[\hat{q}, \hat{p}]] \frac{d^{3}}{d \lambda^{3}}+\frac{1}{3}[\hat{p},[\hat{p}, \hat{q}]] \frac{d^{3}}{d \lambda^{3}}+\frac{1}{24}[\hat{q},[\hat{q},[\hat{q}, \hat{p}]]] \frac{d^{4}}{d \lambda^{4}} \\
& \left.+\frac{1}{8}[\hat{q},[\hat{p},[\hat{p}, \hat{q}]]] \frac{d^{4}}{d \lambda^{4}}-\frac{1}{8}[\hat{p},[\hat{p},[\hat{p}, \hat{q}]]] \frac{d^{4}}{d \lambda^{4}}+\ldots\right\}\left.f(\lambda)\right|_{\lambda=0} G .
\end{aligned}
$$

Expanding out the exponential on the r.h.s. of eq. (3.35) leads to an expression in terms of $f(\hat{p})$ and repeated commutators of $\hat{p}$ and $\hat{q}$. For example, treating the simple commutator as first order, the double commutators as second order and so on, expanding up to second order we get

$$
\begin{aligned}
f(\hat{q}) G=\{f(\hat{p})- & \frac{1}{2} f^{\prime \prime}(\hat{p})[\hat{p}, \hat{q}]+\frac{1}{6} f^{\prime \prime \prime}(\hat{p})[\hat{q},[\hat{q}, \hat{p}]] \\
& \left.\left.+\frac{1}{3} f^{\prime \prime \prime}(\hat{p})[\hat{p},[\hat{p}, \hat{q}]]+\frac{1}{8} f^{\prime \prime \prime \prime}(\hat{p})[\hat{p}, \hat{q}]^{2}+\ldots\right)\right\} G .
\end{aligned}
$$

This is the main result of this section. In order to show how it can be used in practice, in the next subsection we will use it to reproduce the next-to-next-to-leading order result eq. (3.6).

\subsection{Running coupling corrections to NNLO}

In order to compute running-coupling corrections to the duality relation between $\chi_{0}$ and the expansion eq. (3.1) of $\gamma$ in powers of $\alpha_{s}$ at fixed $\alpha_{s} / N$ we use eq. (3.36) with the identification eq. (3.28) of $\hat{p}$ and $\hat{q}$. Furthermore, we assume that $\chi$ is given by its leading order expression,

$$
\chi\left(\hat{\alpha}_{s}, M\right)=\hat{\alpha}_{s} \chi_{0}(M),
$$


so $\hat{q}=\chi_{0}(M)$. The relevant commutators are then

$$
\begin{aligned}
& {[\hat{p}, \hat{q}]=\left[\hat{\alpha}_{s}^{-1} N, \chi_{0}\right]=-N \beta_{0} \chi_{0}^{\prime}(M)} \\
& {[\hat{p},[\hat{p}, \hat{q}]]=\left[\hat{\alpha}_{s}^{-1} N,\left[\hat{\alpha}_{s}^{-1} N, \chi_{0}(M)\right]\right]=\left(N \beta_{0}\right)^{2} \chi_{0}^{\prime \prime}(M)} \\
& {[\hat{q},[\hat{p}, \hat{q}]]=\left[\chi_{0}(M),\left[\hat{\alpha}_{s}^{-1} N, \chi_{0}(M)\right]\right]=0,}
\end{aligned}
$$

where $\chi_{0}$ and its derivatives are all functions of $M$.

We now choose as function $f(\hat{q})$ the function $\gamma_{s}$, which is related to $\chi_{0}$ by naive fixed-coupling duality eq. (3.4), so

$$
M=\gamma_{s}\left(\chi_{0}(M)\right)
$$

Substituting in eq. (3.36) we get

$$
\begin{gathered}
M=\left\{\gamma_{s}\left(\hat{\alpha}_{s}^{-1} N\right)-\frac{1}{2} \gamma_{s}^{\prime \prime}\left(\hat{\alpha}_{s}^{-1} N\right)\left(-N \beta_{0} \chi_{0}^{\prime}(M)\right)+\frac{1}{3} \gamma_{s}^{\prime \prime \prime}\left(\hat{\alpha}_{s}^{-1} N\right)\left(\left(N \beta_{0}\right)^{2} \chi_{0}^{\prime \prime}(M)\right)\right. \\
\left.+\frac{1}{8} \gamma_{s}^{\prime \prime \prime \prime}\left(\hat{\alpha}_{s}^{-1} N\right)\left(-N \beta_{0} \chi_{0}^{\prime}(M)\right)^{2}+O\left(\hat{\alpha}^{3}\right)\right\} G
\end{gathered}
$$

where the prime denotes differentiation with respect to $M$ of $\chi_{0}$ and with respect to $\hat{\alpha}_{s}^{-1} N$ of $\gamma_{s}$. Note that in this equation the first term on the r.h.s. is $O\left(\hat{\alpha}_{s}^{0}\right)$, the second term (coming from the simple commutator in eq. (3.36)) is $O(N)=O\left(\hat{\alpha}_{s}\right)$ in an expansion in powers of $\hat{\alpha}_{s}$ at fixed $\hat{\alpha}_{s}^{-1} N$, and the last two terms, coming from the double commutator and the commutator square, are $O\left(N^{2}\right)=O\left(\hat{\alpha}_{s}^{2}\right)$.

The right-hand side of eq. (3.42) does not yet give the sought-for expression for $\gamma$, because of the residual dependence on $M$ through $\chi_{0}$ and its derivatives. This dependence can be eliminated iteratively by solving the equation to leading order in $\hat{\alpha}_{s}$, back-substituting the result to determine the NLO solution and so on: to LO of course we simply get

$$
M G=\left\{\gamma_{s}\left(\hat{\alpha}_{s}^{-1} N\right)+O\left(\hat{\alpha}_{s}\right)\right\} G,
$$

which replaced in the $O\left(\hat{\alpha}_{s}^{2}\right)$ term gives

$$
M G=\left\{\gamma_{s}\left(\hat{\alpha}_{s}^{-1} N\right)+\frac{1}{2} N \beta_{0} \gamma_{s}^{\prime \prime}\left(\hat{\alpha}_{s}^{-1} N\right) \chi_{0}^{\prime}\left[\gamma_{s}\left(\hat{\alpha}_{s}^{-1} N\right)\right]+O\left(\hat{\alpha}_{s}^{2}\right)\right\} G .
$$

Beyond $O\left(\hat{\alpha}_{s}^{2}\right)$ however the back-substitution becomes nontrivial, because an operator function $\hat{\mathcal{O}}(M)$ on a physical solution on which $M=\gamma_{s}\left(\hat{\alpha}_{s}^{-1} N\right)$ does not simply become $\mathcal{O}\left(\gamma_{s}\left(\hat{\alpha}_{s}^{-1} N\right)\right)$, for precisely the same reason why the functions $f$ and $g$ in eq. (3.27) are different. Specifically, to $O\left(\hat{\alpha}_{s}^{2}\right)$ the back-substitution of the leading-order result into the $O\left(\hat{\alpha}_{s}\right)$ term in eq. (3.42) requires the evaluation of

$$
\chi_{0}^{\prime}(M) G=\left\{\chi_{0}^{\prime}\left[\gamma_{s}\left(\hat{\alpha}_{s}^{-1} N\right)\right]+\frac{1}{2} N \beta_{0} \gamma_{s}^{\prime}\left(\hat{\alpha}_{s}^{-1} N\right) \chi_{0}^{\prime \prime \prime}\left[\gamma_{s}\left(\hat{\alpha}_{s}^{-1} N\right)\right]+O\left(\hat{\alpha}_{s}^{2}\right)\right\} G,
$$

where the result follows using eq. (3.35) again, but with $\hat{p}$ and $\hat{q}$ given by eq. (3.29), and $f=\chi_{0}^{\prime}$. Note that it is sufficient to evaluate eq. (3.45) to $O\left(\hat{\alpha}_{s}^{2}\right)$ because the term on the left-hand side appears in a contribution to eq. (3.42) which is already $O\left(\hat{\alpha}_{s}\right)$. Using this 
result, the back-substitution of the next-to-leading order result eq. (3.44) into the $O\left(\hat{\alpha}_{s}\right)$ term in eq. (3.42) has the form

$$
\begin{aligned}
\frac{1}{2} N \beta_{0} \gamma_{s}^{\prime \prime} \chi_{0}^{\prime}(M) G=\left\{\frac{1}{2} N \beta_{0}\right. & \gamma_{s}^{\prime \prime}\left[\chi_{0}^{\prime}\left(\gamma_{s}\right)+\frac{1}{2} N \beta_{0} \gamma_{s}^{\prime} \chi_{0}^{\prime \prime \prime}\left(\gamma_{s}\right)\right. \\
& \left.\left.+\chi_{0}^{\prime \prime}\left(\gamma_{s}\right) \frac{1}{2} N \beta_{0} \gamma_{s}^{\prime \prime} \chi_{0}^{\prime}\left(\gamma_{s}\right)\right]+O\left(\hat{\alpha}_{s}^{3}\right)\right\} G
\end{aligned}
$$

where the second term in square brackets comes from eq. (3.45), and the third from the expansion of the argument of $\chi^{\prime}$ about $\gamma_{s}$. All terms on the right hand side of this equation now depend on $\hat{\alpha}_{s}^{-1} N$ through $\gamma_{s}$ and its derivatives.

Collecting everything, the final result for $\gamma$ is given by the r.h.s. of eq. (3.42), with the $O\left(\hat{\alpha}_{s}\right)$ term evaluated using the next-to-leading back-substitution eq. (3.46), and the two $O\left(\hat{\alpha}_{s}^{2}\right)$ term evaluated with the leading back-substitution eq. (3.43), to give

$$
\begin{gathered}
M G=\left\{\gamma_{s}+N \beta_{0} \frac{1}{2} \gamma_{s}^{\prime \prime} \chi_{0}^{\prime}\left(\gamma_{s}\right)+\left(N \beta_{0}\right)^{2}\left(\frac{1}{4}\left(\gamma_{s}^{\prime \prime}\right)^{2} \chi_{0}^{\prime}\left(\gamma_{s}\right) \chi_{0}^{\prime \prime}\left(\gamma_{s}\right)+\frac{1}{4} \gamma_{s}^{\prime} \gamma_{s}^{\prime \prime} \chi_{0}^{\prime \prime \prime}\left(\gamma_{s}\right)\right.\right. \\
\left.\left.+\frac{1}{3} \gamma_{s}^{\prime \prime \prime} \chi_{0}^{\prime \prime}\left(\gamma_{s}\right)+\frac{1}{8} \gamma_{s}^{\prime \prime \prime \prime}\left(\chi_{0}^{\prime}\left(\gamma_{s}\right)\right)^{2}\right)+O\left(\hat{\alpha}_{s}^{3}\right)\right\} G .
\end{gathered}
$$

In this way, all terms in eq. (3.47) depend on $\hat{\alpha}_{s}^{-1} N$ through $\gamma_{s}$ and its derivatives, while the dependence on $M$ has been eliminated. The result can be further rewritten entirely in terms of $\chi_{0}$ and its derivatives, or in terms of $\gamma_{s}$ and its derivatives, by exploiting the fact that $\gamma_{s}$ and $\chi_{0}$ and their derivatives are related by the fixed coupling duality relation eq. (3.41) and the following relations which may be obtained differentiating it:

$$
\begin{aligned}
\gamma_{s}^{\prime} & =1 / \chi_{0}^{\prime}, \\
\gamma_{s}^{\prime \prime} & =-\chi_{0}^{\prime \prime} / \chi_{0}^{\prime 3} \\
\gamma_{s}^{\prime \prime \prime} & =\left(3 \chi_{0}^{\prime \prime 2}-\chi_{0}^{\prime} \chi_{0}^{\prime \prime \prime}\right) / \chi_{0}^{\prime 5} \\
\gamma_{s}^{\prime \prime \prime \prime} & =-\left(15 \chi_{0}^{\prime \prime 3}-10 \chi_{0}^{\prime} \chi_{0}^{\prime \prime} \chi_{0}^{\prime \prime \prime}+\chi_{0}^{\prime 2} \chi_{0}^{\prime \prime \prime \prime} \chi_{0}^{\prime \prime}\right) / \chi_{0}^{\prime 7},
\end{aligned}
$$

where $\chi_{0}$ and its derivatives are evaluated as a function of $\gamma_{s}\left(\hat{\alpha}_{s}^{-1} N\right)\left(\right.$ so $\left.\chi_{0}=\hat{\alpha}_{s}^{-1} N\right)$. With these substitutions, eq. (3.47) takes the form

$$
M G=\left\{\gamma_{s}\left(\hat{\alpha}_{s}^{-1} N\right)+\hat{\alpha} \beta_{0} \Delta \gamma_{s s}\left(\hat{\alpha}_{s}^{-1} N\right)+\left(\hat{\alpha} \beta_{0}\right)^{2} \Delta \gamma_{s s s}\left(\hat{\alpha}_{s}^{-1} N\right)+O\left(\hat{\alpha}_{s}^{3}\right)\right\} G,
$$

with $\Delta \gamma_{s s}$ and $\Delta \gamma_{s s s}$ given by eq. (3.5) and eq. (3.6) respectively, thus reproducing the result of ref. $[8,10]$. It is easy to extend this calculation to higher orders, and also to include the contributions due to higher-order terms in the $\beta$ function, which will simply modify the form of the basic commutator eq. (2.14). A systematic investigation of such terms is presented elsewhere $[12,13]{ }^{1}$

1 When going to yet higher orders two further subtleties appear. First, eq. (3.36) cannot be used directly, but rather one must use the modified form of it which has the function $f(\hat{p})$ all the way to the right in all terms. This is due to the fact that it is only when acting on the physical solutions on the right that one can let $\hat{p}=\hat{q}$ iteratively. The second is that when expanding the argument of $\chi_{0}$ and its derivative about $\gamma_{s}$ it must be taken into account that $\gamma_{s}$ is shifted by an amount that does not commute with $\gamma_{s}$ itself, so the Baker-Campbell-Hausdorff formula for the function of the sum of noncommuting operators must be used. 


\section{BFKL leading singularities from GLAP anomalous dimensions}

Let us now turn to the inverse problem to that which we discussed in the previous section: namely, the construction of a BFKL kernel which is dual order by order to a given GLAP anomalous dimension eq. (2.6). Obviously, at the fixed coupling level, duality maps the expansion eq (2.6) of $\gamma$ in powers of $\alpha_{s}$ at fixed $N$ onto the expansion of $\chi$ in powers of $\alpha_{s}$ at fixed $\alpha_{s} / M$ :

$$
\chi\left(\alpha_{s}, M\right)=\chi_{s}\left(M / \alpha_{s}\right)+\alpha_{s} \chi_{s s}\left(M / \alpha_{s}\right)+\ldots,
$$

so that the dual of $\gamma$ when terms up to $\gamma_{n}$ are included in eq. (2.6) is given by $\chi$ with terms up to $\chi_{s^{n}}$ included in eq. (4.1). Now, at the running coupling level, the very definition of $\chi_{s}$ requires an operator ordering, since $\hat{\alpha}_{s}$ and $M^{-1}$ do not commute. Moreover, eq. (3.12) implies that different operator orderings within $\chi_{s}$ differ by terms which are of the same order as $\chi_{s}$ itself (and similarly to subsequent orders). Hence, as already mentioned in section 2, the determination of the dual $\chi_{s}$ involves the computation of an infinite set of running coupling corrections to duality. Running duality contributions to $\chi_{s}$ may be computed to any desired order by using the method of the previous section, but with the identification eq. (3.29) of $\hat{p}, \hat{q}$. This may be useful for the determination $[13,14]$ of an approximate form of $\chi_{n}$ beyond the known next-to-leading order $\chi_{1}$. However, in this section we will show that, by choosing a suitable operator ordering, it is in fact possible to determine runnig coupling corrections to all orders in $\alpha_{s}$, and we will construct explicitly the running coupling dual to $\gamma$ eq. (2.6) up to next-to-leading order.

\subsection{Operator ordering and leading order}

In order to introduce our proof, consider first a simplified case. Namely, assume that we start with the model leading-order GLAP anomalous dimension

$$
\gamma_{\text {toy }}=\alpha_{s}(t)\left(N^{-1}-1\right) .
$$

This anomalous dimension has the same small $-N$ behaviour as the leading-order GLAP anomalous dimension (up to an overall numerical factor, which is irrelevant here), and it respects momentum conservation in that it vanishes at $N=1$. In fact, the simple form eq. (4.2) turns out to be a surprisingly good approximation to the full leading-order GLAP anomalous dimension, even though this is immaterial for our purposes. We wish to determine the $\chi_{s}$ kernel which is dual to it. Because the anomalous dimension is very simple, we can solve all the relevant evolution equations exactly.

At the fixed coupling level, the dual eq. (2.8) of $\gamma_{\text {toy }}$ is given by

$$
\chi_{\text {toy }}\left(M / \alpha_{s}\right)=\frac{\alpha_{s}}{\alpha_{s}+M}=\frac{1}{1+M / \alpha_{s}} .
$$

We proceed by trial and error: we guess a particular ordering in $\chi$, we determine the corresponding $\gamma$ anomalous dimension explicitly, and we compare the result to eq. (4.2). Specifically, we make the ansatz

$$
\chi_{\text {toy }}\left(\hat{\alpha}_{s}^{-1} M\right) \equiv \frac{1}{1+\hat{\alpha}_{s}^{-1} M}=\frac{1}{1+M \hat{\alpha}_{s}^{-1}-\beta_{0}},
$$


where in the last step we used the commutator eq. (2.14). This can be thought of as the result of writing the fixed-coupling dual (4.3) as a function of $\alpha_{s}^{-1} M$, and then letting $\alpha_{s} \rightarrow \hat{\alpha}_{s}$ with this particular ordering. Substituting in the running-coupling BFKL equation (2.17) we get

$$
\left(1-\beta_{0}+M \hat{\alpha}_{s}^{-1}\right) N G(N, M)=\tilde{G}_{0}(M)+G(N, M),
$$

where $\tilde{G}_{0}(M)=\left(1-\beta_{0}+M \hat{\alpha}_{s}^{-1}\right) G(N, M)$ is a modified boundary condition. We now assume that $\hat{\alpha}_{s}$ takes the leading-order form eq. (2.13). As proven in Ref. [9], the large $Q^{2}$ behaviour of the solution to eq. (4.5) is the same as that of the solution of the associated homogenous equation, multiplied by a $Q^{2}$-independent boundary condition. Hence, we determine it setting $\tilde{G}=0$ in Eq. (4.5), which then has solution

$$
G(N, M)=M^{\beta_{0}^{-1}\left(1-N^{-1}\right)-1} e^{M / \beta_{0} \alpha_{s}} .
$$

In order to determine the large $Q^{2}$ behaviour explicitly, we must invert the $M$-Mellin transform:

$$
\begin{aligned}
G(N, t) & =\int \frac{d M}{2 \pi i} M^{\beta_{0}^{-1}\left(1-N^{-1}\right)-1} e^{M / \beta_{0} \alpha_{s}} e^{M t} \\
& =\left(\beta_{0} \alpha_{s}(t)\right)^{\beta_{0}^{-1}\left(1-N^{-1}\right)}\left[\Gamma\left(\beta_{0}^{-1}\left(1-N^{-1}\right)-1\right)\right]^{-1}
\end{aligned} .
$$

The anomalous dimension is then

$$
\gamma\left(N, \alpha_{s}(t)\right) \equiv \frac{\partial}{\partial t} \ln G(N, t)=\alpha_{s}(t)\left(N^{-1}-1\right)
$$

which coincides with eq. (4.2). Hence, we find the surprizing result that for the particular ordering (4.4) the running-coupling dual coincides exactly with the naive dual.

Let us now prove that this result holds in general at the leading $\ln Q^{2}$ level, provided the operator ordering of eq. (4.4) is adopted. As discussed in sect. 3.2, the dual BFKL kernel is constructed by starting with the pole condition eq. (3.17), and determining the operator $\chi\left(\hat{\alpha}_{s}, M\right)$ which satisfies eq. (3.20). Now, at leading order the pole condition is just

$$
\hat{\alpha}_{s}^{-1} M G(N, M)=\gamma_{0}(N) G(N, M) .
$$

Therefore, given the ordinary inverse function $\chi_{s}$

$$
\chi_{s}\left(\gamma_{0}(N)\right)=N
$$

then the operator-valued function $\chi_{s}\left(\hat{\alpha}_{s}^{-1} M\right)$, when acting on the solution $G$, using repeatedly eq. (4.9) is seen to satisfy

$$
N G(N, M)=\chi_{s}\left(\hat{\alpha}_{s}^{-1} M\right) G(N, M)
$$

Namely, each time $\hat{\alpha}_{s}^{-1} M$ acts on the physical state $G(N, M)$ it gives $\gamma(N)$, so acting repeatedly gives powers of the commuting operator $\gamma(N)$. Therefore if $\chi_{s}$ is chosen to satisfy the inverse function condition eq. (4.10) the desired dual BFKL equation eq. (4.11) immediately follows, which is what we set out to prove. Note that $\left(\hat{\alpha}_{s}^{-1} M\right)^{-1}=\hat{\alpha}_{s} M^{-1}$, so we can equivalently take $\chi_{s}$ to be a function of $\hat{\alpha}_{s} M^{-1}$. 


\subsection{Next-to-leading corrections}

The result of the previous subsection can be derived equivalently by letting

$$
\begin{aligned}
& \hat{p}=\hat{\alpha}_{s}^{-1} M \\
& \hat{q}=\hat{\alpha}_{s}^{-1} \gamma\left(\hat{\alpha}_{s}, N\right),
\end{aligned}
$$

so that when $\gamma\left(\hat{\alpha}_{s}, N\right)=\hat{\alpha}_{s} \gamma_{0}(N)$, then $\hat{p}$ and $\hat{q}$ commute, and eq. (3.36) implies that $f(\hat{p}) G=f(\hat{q}) G$ so taking $f \equiv \chi_{s}$ defined by naive duality (4.10) we get

$$
\chi_{s}(\hat{p}) G(N, M)=\chi_{s}\left(\gamma_{0}(N)\right) G(N, M),
$$

whence the result. However, at next-to-leading order $\hat{q}=\gamma_{0}(N)+\hat{\alpha}_{s} \gamma_{1}(N)$, and $[\hat{p}, \hat{q}]=$ $-\beta_{0} \hat{\alpha}_{s} \gamma_{1}(N)$, which is of the same order as $\gamma_{1}(N)$. Likewise, all higher order commutators $[\hat{p},[\hat{p}, \ldots,[\hat{p}, \hat{q}]]]$ are of the same order $\hat{\alpha}_{s}$. Therefore, the iterative commutator approach of the previous section does not help and we must proceed to all orders.

This can be done by noting that the desired next-to-leading order contribution $\chi_{s s}\left(\hat{\alpha}_{s}^{-1} M\right)$ to the BFKL kernel must satisfy [recall eq. (3.19)]

$$
\left[\gamma_{0}\left(\chi_{s}(\hat{p})+\hat{\alpha}_{s} \chi_{s s}(\hat{p})\right)+\hat{\alpha}_{s} \gamma_{1}\left(\chi_{s}(\hat{p})\right)\right] G(N, M)=\hat{p} G(N, M),
$$

where $\hat{p}$ is given by eq. (4.12). At leading order, this just gives

$$
\gamma_{0}\left(\chi_{s}(\hat{p})\right) G(N, M)=\hat{p} G(N, M),
$$

which is equivalent to eq. (4.13). In order to determine the next-to-leading order $\chi_{s s}$ it is necessary to expand out the argument of $\gamma_{0}$ in eq. (4.14), which is nontrivial because $\left[\hat{\alpha}_{s}, \chi_{s s}(\hat{p})\right] \neq 0$. It turns out, however, that this commutator can be determined explicitly, and this is sufficient to solve eq. (4.14) by expansion.

To this end, write $\chi_{s s}$ as a power series:

$$
\chi_{s s}(\hat{p})=\sum_{i} k_{i} \hat{p}^{-i} .
$$

In practice we will find that only positive powers of $i$ contribute to the sum (since $\chi_{s s}$ vanishes when $\hat{\alpha}_{s} \rightarrow 0$ ), but this is not necessary for our argument. Hence, we wish to determine the commutator $\left[\hat{p}^{-n}, \hat{\alpha}_{s}\right]$. To this purpose, first we note that the commutator eq. (3.12) implies

$$
\left[\hat{\alpha}_{s}, \hat{p}^{-1}\right]=-\beta_{0} \hat{p}^{-1} \hat{\alpha}_{s} \hat{p}^{-1},
$$

which can be rewritten as

$$
\hat{p}^{-1} \hat{\alpha}_{s}=\hat{\alpha}_{s} \hat{p}^{-1}\left(1-\beta_{0} \hat{p}^{-1}\right)^{-1}=\hat{\alpha}_{s}\left(\hat{p}-\beta_{0}\right)^{-1} .
$$

Iterating this equation, it immediately follows that

$$
\hat{p}^{-n} \hat{\alpha}_{s}=\hat{\alpha}_{s}\left(\hat{p}-\beta_{0}\right)^{-n} .
$$


Similarly,

$$
\left[\hat{\alpha}_{s}, \hat{p}\right]=\beta_{0} \hat{\alpha}_{s}
$$

implies

$$
\hat{p}^{n} \hat{\alpha}_{s}=\hat{\alpha}_{s} \hat{p}^{n}\left(1-\beta_{0} \hat{p}^{-1}\right)^{n}=\hat{\alpha}_{s}\left(\hat{p}-\beta_{0}\right)^{n},
$$

or, equivalently, eq. (4.19) holds with both positive and negative $n$. Using this in the expansion eq. (4.16) of $\chi_{s s}$ implies that

$$
\chi(\hat{p}) \hat{\alpha}_{s}=\hat{\alpha}_{s} \chi\left(\hat{p}-\beta_{0}\right) .
$$

Of course, this result holds for any function that may be expanded as a series of powers of $\hat{p}$.

It is now easy to determine the function on the left-hand side of eq. (4.14), i.e. $\gamma_{0}\left(\chi_{s}(\hat{p})+\hat{\alpha}_{s} \chi_{s s}(\hat{p})\right)$, by expanding

$$
\gamma_{0}(N)=\sum_{j} g_{j} N^{j}
$$

where in practice of course the sum runs over $-1 \leq j \leq \infty$, though again we will not actually use this in our argument. We must therefore evaluate

$$
\left(\chi_{s}+\hat{\alpha}_{s} \chi_{s s}\right)^{n}=\chi_{s}^{n}+\left(\chi_{s}^{n-1} \hat{\alpha}_{s} \chi_{s s}+\chi_{s}^{n-2} \hat{\alpha}_{s} \chi_{s s} \chi_{s}+\ldots+\hat{\alpha}_{s} \chi_{s s} \chi_{s}^{n-1}\right)+O\left(\hat{\alpha}_{s}^{2}\right),
$$

where $\chi_{s}$ and $\chi_{s s}$ are both functions of $\hat{p}$. Using eq. (4.22) we get

$$
\left(\chi_{s}+\hat{\alpha}_{s} \chi_{s s}\right)^{n}=\chi_{s}^{n}(\hat{p})+\hat{\alpha}_{s} \frac{\chi_{s}^{n}\left(\hat{p}-\beta_{0}\right)-\chi_{s}^{n}(\hat{p})}{\chi_{s}\left(\hat{p}-\beta_{0}\right)-\chi_{s}(\hat{p})} \chi_{s s}(\hat{p})+O\left(\hat{\alpha}_{s}^{2}\right),
$$

where we have made use of the fact that $\left[\chi_{s}\left(\hat{p}-\beta_{0}\right), \chi_{s}(\hat{p})\right]=0$ and we have noticed that for a pair of commuting operators $A$ and $B$,

$$
A^{n-1}+A^{n-2} B+\ldots+B^{n-1}=\frac{A^{n}-B^{n}}{A-B} .
$$

Furthermore, it is easy to see that eq. (4.25) also holds for negative $n$, by expanding

$$
\begin{aligned}
\left(\chi_{s}+\hat{\alpha}_{s} \chi_{s s}\right)^{-n} & =\left(\chi_{s}^{-1}+\chi_{s}^{-1} \hat{\alpha}_{s} \chi_{s s} \chi_{s}^{-1}\right)^{n}+O\left(\hat{\alpha}_{s}^{2}\right) \\
& =\chi_{s}^{-n}+\left(\chi_{s}^{-n} \hat{\alpha}_{s} \chi_{s s} \chi_{s}^{-1}+\chi_{s}^{-(n-1)} \hat{\alpha}_{s} \chi_{s s} \chi_{s}^{-2}+\ldots+\chi_{s}^{-1} \hat{\alpha}_{s} \chi_{s s} \chi_{s}^{-n}\right)+O\left(\hat{\alpha}_{s}^{2}\right),
\end{aligned}
$$

and then using again eq. (4.22) but with $\chi_{s} \rightarrow \chi_{s}^{-1}$.

Substituting eq. (4.25) in the expansion eq. (4.23) of $\gamma$ gives

$$
\gamma_{0}\left(\chi_{s}(\hat{p})+\hat{\alpha}_{s} \chi_{s s}(\hat{p})\right)=\gamma_{0}\left(\chi_{s}(\hat{p})\right)+\hat{\alpha}_{s} \chi_{s s}(\hat{p}) \frac{\gamma_{0}\left(\chi_{s}\left(\hat{p}-\beta_{0}\right)\right)-\gamma_{0}\left(\chi_{s}(\hat{p})\right)}{\chi_{s}\left(\hat{p}-\beta_{0}\right)-\chi_{s}(\hat{p})}
$$


Using this in eq. (4.14) with the leading-order eq. (4.15) we get the remarkably simple result

$$
\chi_{s s}(\hat{p})=\beta_{0}^{-1} \gamma_{1}\left(\chi_{s}(\hat{p})\right)\left(\chi_{s}\left(\hat{p}-\beta_{0}\right)-\chi_{s}(\hat{p})\right) .
$$

This is the running coupling generalization of the well-known fixed coupling result $\chi_{s s}\left(M / \alpha_{s}\right)=-\frac{\gamma_{1}\left(\chi_{s}\left(M / \alpha_{s}\right)\right)}{\gamma_{0}^{\prime}\left(\chi_{s}\left(M / \alpha_{s}\right)\right)}$, to which it reduces in the limit $\beta_{0} \rightarrow 0\left(\right.$ since $\left.\chi_{s}^{\prime}=1 / \gamma_{0}^{\prime}\left(\chi_{s}\right)\right)$.

The result can be generalized to higher orders and systematized by performing the expansion of the higher-order generalizations of operator duality condition eq. (4.14) by means of the Baker-Campbell-Hausdorff formula for the Taylor expansion of a function of the sum of two non-commuting operators.

\section{Conclusions}

In this paper we have presented an explicit constructive proof of the fact that the leadingtwist duality between the BFKL and GLAP equations holds to all orders, with the running of the coupling included to any desired order. Besides its conceptual interest, this result allows one to exploit fully the information contained in fixed-order determinations of the BFKL and GLAP kernels by using each to resum the unresummed singularities in the other.

Because of the instability of the fixed order expansion of the BFKL kernel in powers of $\alpha_{s}$ due to unresummed collinear singularities, our running duality is especially useful in constructing a resummed form of this kernel, using the collinear resummation implicit in the fixed order expansion of the GLAP kernel. The running duality can then be used again to derive from the resummed kernel a corresponding anomalous dimension in which small $x$ singularities are resummed. Indeed, several of the results contained in this paper were used already in the explicit construction of a fully stable resummed perturbative expansion of small $x$ anomalous dimensions $[2,3,6]$. Further investigations of the application of the formalism presented here to this and related problems will be presented elsewhere [13]. 


\section{References}

[1] G. Altarelli, R. D. Ball and S. Forte, Nucl. Phys. B575, 313 (2000); see also hepph/0001157.

[2] G. Altarelli, R. D. Ball and S. Forte, Nucl. Phys. Proc. Suppl. 135 (2004) 163

[3] G. Altarelli, R. D. Ball and S. Forte, CERN-PH-TH-2005-174, hep-ph/0001157.

[4] M. Ciafaloni, D. Colferai and G. P. Salam, Phys. Rev. D 60 (1999) 114036.

[5] M. Ciafaloni, D. Colferai, G. P. Salam and A. M. Stasto, Phys. Rev. D 66 (2002) 054014; Phys. Lett. B 576 (2003) 143; Phys. Rev. D 68 (2003) 114003.

[6] G. Altarelli et al., "Resummation", in M. Dittmar et al., hep-ph/0511119.

[7] R. D. Ball and S. Forte, Phys. Lett. B405 (1997) 317.

[8] R. D. Ball and S. Forte, Phys. Lett. B465 (1999) 271.

[9] G. Altarelli, R. D. Ball and S. Forte, Nucl. Phys. B 621 (2002) 359.

[10] S. Forte and R. D. Ball, AIP Conf. Proc. 602 (2001) 60 hep-ph/0109235.

[11] E. Eriksen, J. Math. Phys.9 (1969) 790

[12] P. Falgari, Laurea Thesis, Milan University, April 2005

[13] R. D. Ball, P. Falgari, S. Forte and S. Marzani, in preparation

[14] S. Marzani, Laurea Thesis, Milan University, April 2005 